\title{
An Alternative Technique for Ascending and Arcus Aorta Repair in Case of Vascular Diameter Mismatch
}

\author{
Bulend Ketenci, MD, Tamer Kehlibar, MD, Abdulkerim Ozhan, MD, Mehmet Yilmaz, MD, \\ Erhan Guler, MD, Canan Karakaya, MD, Mehmet Erdem Memetoglu, MD, Murat Demirtas, MD \\ Department of Cardiovascular Surgery, Dr Siyami Ersek Thoracic and Cardiovascular Surgery Training and Research Hospital, \\ Istanbul, Turkey
}

\section{ABSTRACT}

Complicated Type A intramural hematoma involving the arcus aorta requires emergency correction of the aortic arch. Surgical options include reimplantation of the brachiocephalic vessels as an island to a vascular graft, debranching aortic arch surgery, and Kazui technique. This report describes a modified technique for aortic arch repair in a patient with vascular diameter mismatch between the ascending and descending aorta, as well as an intimal tear between the brachiocephalic vessels.

\section{INTRODUCTION}

Acute aortic syndrome defines a group of life-threatening conditions that include aortic dissection, intramural hematoma, and penetrating aortic ulcer. Intramural hematoma (IMH) is caused by the rupture of a vasa vasorum and the ensuing propagation of blood within the layers of the aortic wall. Spontaneous resolution happens in less than $10 \%$ of IMHs, while the rest may progress into an acute aortic dissection (AD), during the course of the disease [Tsai 2005; Mussa 2016]. A substantial portion of Type A IMHs progress into $\mathrm{AD}$ and survival is poor at 5 years, while long-term prognosis is more favorable in patients where IMH does not progress into $\mathrm{AD}$ [Erbel 2014].

Emergent surgery is indicated in cases complicated with pericardial effusion, periaortic hematoma, or a large-sized aneurysm. However, an initial conservative approach of watchful waiting for clinical progression with consecutive imaging may be a plausible option for patients with high risk comorbidities, especially in the absence of aortic dilatation $(<50 \mathrm{~mm})$ and when IMH thickness is $<11 \mathrm{~mm}$ [Erbel 2014].

\section{CASE STUDY}

A 66-year-old male patient was admitted to the emergency department with chest and back pain of 3-day duration. The

Received August 14, 2019; accepted February 28, 2020.

Correspondence: Abdulkerim Özhan, MD, Tibbiye Cd No 13, Haydarpasa, Uskudar, Istanbul, Turkey (e-mail:draozhan@gmail.com). work-up was negative for myocardial ischemia. Initial chest CT angiography (CTA) revealed a $50 \mathrm{~mm}$ fusiform aneurysm of the ascending aorta (AscA) with a $5 \mathrm{~mm}$ IMH (Figure 1). Arcus aorta diameter was $37 \mathrm{~mm}$ at its widest section, and the descending aorta diameter was $30 \mathrm{~mm}$ at the level of the subclavian artery. Transthoracic echocardiography revealed a $23 \mathrm{~mm}$ aortic annulus, and a $38 \mathrm{~mm}$ sinus valsalva diameter. Ejection fraction was $60 \%$, and no aortic regurgitation was detected. The patient had a $38^{\circ} \mathrm{C}$ fever, methicillin susceptible Staphylococcus epidermidis was identified from blood cultures for which antibiotherapy was started. At the one-week follow up, the patient was hemodynamically stable with no decrease in hematocrit levels nor a recurrent fever. However, control CTA scan showed a marked increase in AscA diameter from $50 \mathrm{~mm}$ to $60 \mathrm{~mm}$, and in IMH thickness from $5 \mathrm{~mm}$ to $11 \mathrm{~mm}$. IMH also had extended from the AscA to the level of renal arteries. There also was a penetrating ulcer on the distal left lateral AscA. Therefore, an emergent surgery for ascending, arcus and descending aorta was planned.

Surgical Technique: The patient was placed on cardiopulmonary bypass through the right axillary artery and right atrium. Arcus aorta and its branches were liberated. Cardiac arrest was obtained by antegrade blood cardioplegia and $25^{\circ} \mathrm{C}$ systemic hypothermia. Cross clamp was placed on the distal AscA. The aortic valve was explored through transverse aortotomy and found to be tricuspid without significant regurgitation. IMH was visualized from the interior between the tunica media and adventitia of the AscA. The AscA was resected and a 30-mm Dacron vascular graft was anastomosed to the proximal AscA in an end-to-end fashion (Figure 2).

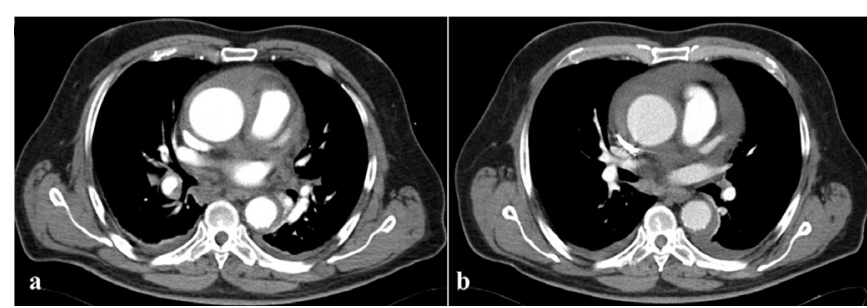

Figure 1. A) Ascending aorta diameter was $50 \mathrm{~mm}$ and intramural hematoma thickness was $5 \mathrm{~mm}$ in computed tomography at the time of admission; B) The control computed tomography showed an increase in intramural hematoma thickness to $11 \mathrm{~mm}$ and ascending aorta diameter to $60 \mathrm{~mm}$. 

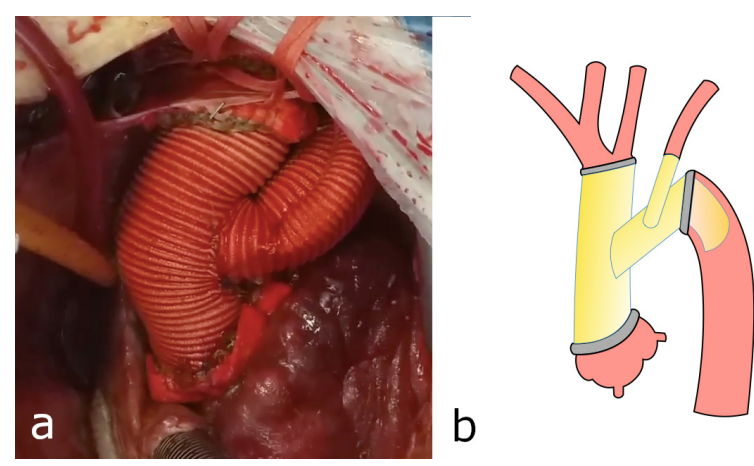

Figure 2. A) Surgical repair of ascending and arcus aorta; B) Illustrative image of the surgical aortic arch reconstruction.

The patient was cooled to $18^{\circ} \mathrm{C}$, and the cross clamp was moved to the innominate artery. Continuous cerebral perfusion was provided through the axillary cannula with the start of deep hypothermic circulatory arrest. An intimal tear was seen between the left common carotid artery (LCCA) and left subclavian artery (LSA). LSA was separated from the arcus aorta. The innominate artery and LCCA ostia were prepared as an island. Another intimal tear was seen on the inner curvature of the arcus aorta extending to the descending aorta. A $26 \mathrm{~mm}$ Dacron graft was prepared as a neo-arcus graft and a portion of the same graft was placed inside the descending aorta as a free-end elephant trunk graft. Thereafter the distal end-to-end anastomosis was carried out between these two grafts and the descending aorta. A $10 \mathrm{~mm}$ Dacron graft was anastomosed to LSA. The distal anastomosis of the $30 \mathrm{~mm}$ Dacron graft was sutured to the island of the innominate and LCCA ostia in an end-to-end fashion. The proximal anastomosis of the $26 \mathrm{~mm}$ Dacron graft was sutured to the $30 \mathrm{~mm}$ graft in an end-to-side fashion. Finally, the proximal end of the $10 \mathrm{~mm}$ graft was connected to the $26 \mathrm{~mm}$ graft in an endto-side fashion. The hypothermic circulatory arrest period was 64 minutes.

Postoperative course: The patient was extubated at the postoperative 20th hour and showed no neurological dysfunction. He was discharged from the hospital on the 10th postoperative day. No adverse events were observed during the 3 months of follow up. The postoperative CT angiography is shown in Figure 3. Further endovascular intervention for the descending aorta was planned in collaboration with interventional radiology.

\section{DISCUSSION}

The definitive therapy options in type A IMH are open surgical repair or hybrid procedures. Open surgical repair is the perfect solution if the disease is localized to the AscA. When the disease extends to the aortic arch and the descending aorta, further endovascular interventions become necessary. Total surgical repair of the AscA and arcus aorta completed by elephant trunk repair is the corrective intervention of choice despite its associated risks [Erbel 2014].

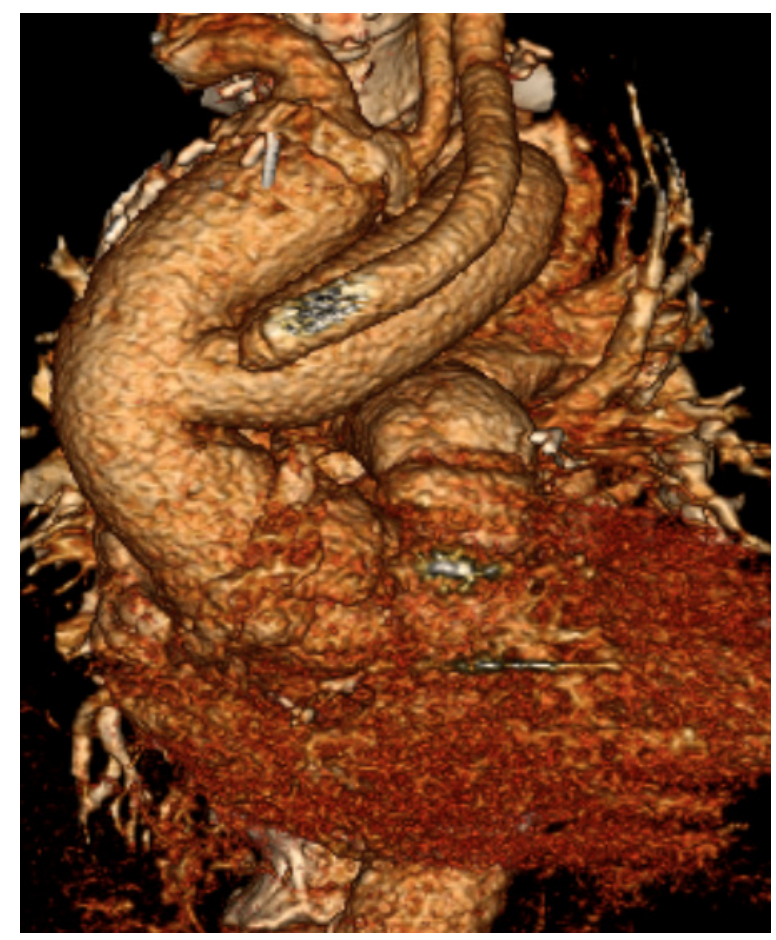

Figure 3. Three-dimensional visualization of the postoperative computed tomography.

Reimplantation of the brachiocephalic vessels as a Carrel patch into a vascular graft and Kazui technique are the most widely used techniques today [Ghanta 2018; Brown 2018].

In our case, the diameter mismatch between the ascending and descending aorta, and the intimal tear locations in the aorta led us to modify the current standard reconstructive surgical repair technique. Classical debranching aortic arch surgery has 8 anastomoses [Ghanta 2018; Brown 2018]. When the ascending aortic graft is anastomosed to the island of aortic arcus branches in an end-to-end fashion, the procedure results in a fewer number of anastomoses in addition to spared time. Reduction in the number of anastomoses may prevent some complications, especially reducing the risk of bleeding from anastomosis sites. Preparation of the arcus branches as a single island may be a good option for anastomosis, but in our case, there was an intimal tear between the LSA and LCCA, therefore, the LSA was separated from the arcus aorta.

The 64-minute hypothermic circulatory arrest period is a considerable time, but this is common in arcus aorta surgery, and the antegrade cerebral perfusion provides sufficient protection.

Although the use of 3 separate grafts may increase the financial burden of the procedure in our technique, the other alternatives such as classical debranching technique and Kazui technique are not cheaper and reliable, in terms of the special conditions of our patient [Ghanta 2018; Brown 2018].

The mismatch between the diameter of the ascending and descending aorta may result in an inappropriate anastomosis. The use of 2 grafts of different sizes may provide better diameter compatibility between the ascending and descending aorta. 
Vascular kinking is likely to occur with the use of a single graft, when constructing the ascending and the arcus aorta [Velayudhan 2017]. "Two grafts" method also prevents vascular kinking while protecting against graft diameter mismatch.

We successfully applied this method in a single patient with conditions requiring a modified technique. We believe this technique can be preferred in aortic surgery cases with aortic diameter mismatch and a vascular angulation between the ascending and descending aorta.

\section{REFERENCES}

Brown CR, Bavaria JE, Desai ND, et al. 2018. Ascending and arch aortic aneurysms. In: Cohn LH, Adams DH, eds. Cardiac Surgery in the Adult. 5th ed. New York, NY: McGraw-Hill; 1037-74.

Erbel R, Aboyans V, Boileau C, et al. 2014. ESC Guidelines on the diagnosis and treatment of aortic diseases. Eur Heart J 35:2873-926.

Ghanta RK, Mery CM, Kron IL, et al. 2018. Aortic dissection. In: Cohn LH, Adams DH, eds. Cardiac Surgery in the Adult. 5th ed. New York, NY: McGraw-Hill 1003-35.

Mussa FF, Horton JD, Moridzadeh R, et al. 2016. Acute aortic dissection and intramural hematoma. JAMA 316:754-63.

Tsai TT, Nienaber CA, Eagle KA, et al. 2005. Acute aortic syndromes. Circulation 112:3802-13.

Velayudhan BV, Idhrees AM, et al. 2017. A new technique for graftto-graft anastomosis to reconstruct the aortic arch. Ann Thorac Surg $103: e 563-4$. 